\title{
Probabilistic Dressing of a Storm Surge Prediction in the Adriatic Sea
}

\author{
R. Mel ${ }^{1}$ and P. Lionello ${ }^{2}$ \\ ${ }^{1}$ Dipartimento di Ingegneria Civile, Edile ed Ambientale (DICEA), Università degli Studi di Padova, Via Marzolo 9 , \\ 35131 Padova, Italy \\ ${ }^{2}$ Dipartimento Scienze e Tecnologie Biologiche e Ambientali (DISTEBA), Università del Salento, Via Tancredi 7, 73100 Lecce, Italy \\ Correspondence should be addressed to R. Mel; riccardo.mel@dicea.unipd.it
}

Received 27 November 2015; Revised 12 April 2016; Accepted 8 May 2016

Academic Editor: Lars R. Hole

Copyright (C) 2016 R. Mel and P. Lionello. This is an open access article distributed under the Creative Commons Attribution License, which permits unrestricted use, distribution, and reproduction in any medium, provided the original work is properly cited.

Providing a reliable, accurate, and fully informative storm surge forecast is of paramount importance for managing the hazards threatening coastal environments. Specifically, a reliable probabilistic forecast is crucial for the management of the movable barriers that are planned to become operational in 2018 for the protection of Venice and its lagoon. However, a probabilistic forecast requires multiple simulations and a considerable computational time, which makes it expensive in real-time applications. This paper describes the ensemble dressing method, a cheap operational flood prediction system that includes information about the uncertainty of the ensemble members by computing it directly from the meteorological input and the local spread distribution, without requiring multiple forecasts. Here, a sophisticated error distribution form is developed, which includes the superposition of the uncertainty caused by inaccuracies of the ensemble prediction system, which depends on surge level and lead time, and the uncertainty of the meteorological forcing, which is described using a combination of cross-basin pressure gradients. The ensemble dressing is validated over a 3-month-long period in the year 2010, during which an exceptional sequence of storm surges occurred. Results demonstrate that this computationally cheap method can provide an acceptably realistic estimate of the uncertainty.

\section{Introduction}

Increasing population, tourism pressure, sea level (SL) rise, and increased storminess [1] pose a significant hazard for many coastal areas of the world. The generation of extreme coastal SLs and wind-waves produces overtopping of flood defenses and constitutes a significant threat to life and property, becoming a hazardous threat for coastal communities. Providing a fully informative forecast of storm surge and of associated flooding is of paramount importance for a wide range of problems related to coastal environments protection, receiving increasing attention from both the research and the operational communities. In fact, in the last decades, several countries have developed technologically advanced storm surges forecast systems $[2,3]$.

Venice Lagoon, Italy, which is the subject of the current study, is located at the shore of the Northern Adriatic Sea. Floods, which are locally called "acqua alta" (meaning "high water"), are a recurrent threat for this unique city, damaging monuments and buildings, frequently disrupting everyday life and affecting the local economy.

The dynamics of floods in Venice and other islands of the lagoon have been described by several studies (e.g., [4-6]). The morphology of the Adriatic Sea, shallow in its northern part and deep in the south, and the basin's shape, elongated, semienclosed, and surrounded by mountain chains, exposes the Venice Lagoon to intense storm surges, which are caused by cyclones moving along the North Atlantic storm track or secondary cyclones triggered by these systems in the northwestern MR [5, 7].

Because of catastrophic past episodes (1966, 1979, and 1986), increased frequency caused by mean SL rise in the past decades, and concern for future further intensification [8], a tide forecast centre was established by the Venice Municipality in 1980 (ICPSM, Istituzione Centro Previsioni e Segnalazioni Maree), which delivers the SL prediction using 
a set of statistical and hydrodynamic numerical models [9]. In the near future, an accurate forecast will be required also for supporting the management of the projected mobile closure gates that are presently being built across the three inlets that connect the Venice Lagoon with the Adriatic Sea (called MOSE, Modulo Sperimentale Elettromeccanico, experimental electric-mechanic module).

Accuracy of SL prediction depends on the quality of the model, of the meteorological forcing (generally provided by an independent weather prediction centre), and of the initial condition from which the prediction starts. In order to reduce errors in the latter, forecast systems recently include a data assimilation procedure, which can be based on adjoint models [10] or on Kalman filters [11].

Though a wide set of models has successfully provided adequate storm surge predictions for many years, they contain unavoidable imprecision due to inaccurate forcing by atmospheric pressure and wind, surge initial condition, the simplifications and finite resolution of the model itself, and the form of the coupling between atmospheric winds and the ocean surface. These uncertainties are important to be understood and described for many practical applications, such as in the field of risk analysis and decisionmaking, of which the MOSE barriers' management is a clear example. Without incorporating all sources of uncertainty adequately, forecasts can be probabilistically unreliable (e.g., overconfident), because it is difficult to assess the situationspecific risk of a particular water level being exceeded. In the last decades, the need to integrate model predictions with an estimate of uncertainty has been pointed out by many authors (see, e.g., the early contribution of Beven [12] and the more recent studies $[13,14])$. Forecast systems of storm surges need to deliver not only a prediction with the highest precision, but also information on uncertainty of the forecast and on probability to cross critical SL thresholds. This kind of forecast can be produced by the ensemble prediction system (EPS) technique. EPS assesses uncertainty in forecasts by running not one but several different realizations, which may differ because they use slightly different initial conditions, boundary conditions, and model physics.

Flowerdew et al. [15] were the first to describe an application of the EPS in operational surge prediction system, and, recently, EPS storm surge forecast systems have started to appear in operational centres [16]. Recently, Mel and Lionello $[17,18]$ have shown the advantages of a probabilistic forecast with respect to the more common high resolution forecast (deterministic forecast, DF) for predicting storm surge in the Northern Adriatic Sea. Their results describe EPS as an approach that can deliver an accurate estimate of the probability of exceeding a specified critical level during the forecast time window and, in general, it can compute the uncertainty in the timing of the peak and magnitude of the surge. However, the EPS approach requires a considerable computational cost for real-time application; thus there have been many attempts to offer solutions in different contexts $[19,20]$. The most efficient procedure is the ensemble dressing forecast (EDF), widely used in hydrology and meteorology [21-23].

The utility of a simply dressed forecast in storm surge prediction for the city of Venice was already described

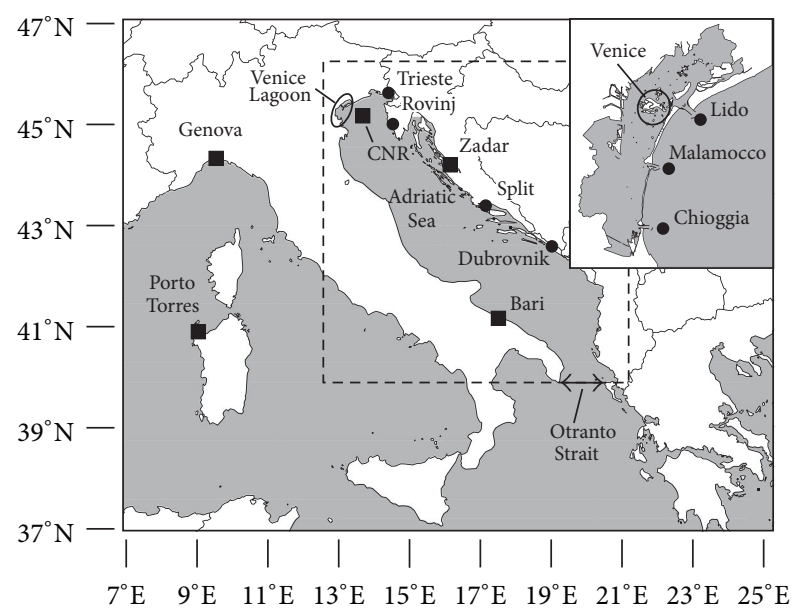

FIgUre 1: Latitude and longitude of Italy, the Adriatic Sea, and the Venice Lagoon are reported with the HYPSE domain. The locations of the tide and pressure gauges used in this study are also shown: the three inlet-gates of the Venice Lagoon, Trieste, Rovinj, Split, and Dubrovnik tide gauges and CNR platform, Genova, Porto Torres, Zadar, and Bari pressure gauges.

in $[18,24]$. In these previous studies, authors have shown the feasibility of dressing a deterministic forecast with an estimate of its uncertainty, which avoids carrying out multiple simulations. This study further continues this development, by expanding the analysis of the improvement of the Brier score over five different gauges in the Adriatic Sea, including explicitly the uncertainty of the EPS system and considering three full months and not only 10 events as in [24]. Further, more parameters than before have been used for the computation of the uncertainty, which has been related not only to the uncertainty of the meteorological fields but also to the inaccuracies of the EPS surge forecast, depending on the gauge, surge magnitude, and lead time.

This paper is organized as follows. In Section 2, we briefly recall the hydrodynamic model and describe previous applications of EPS in the Venice Lagoon. The description of four simplified methods to estimate the uncertainty and the results is then presented in Section 3, comparing the uncertainties provided between different kinds of dressing and the EPS, respectively. A set of conclusions closes the paper.

\section{Methods and Data}

In this study, the EPS method is first applied to the Adriatic Sea during a 3-month-long period in the year 2010.

Tide gauge observations, provided every hour, supply direct evaluation of the final water level at the gauges of Trieste, Lido Diga Sud, Malamocco Diga Nord, Chioggia Diga Sud, Rovinj, Split, and Dubrovnik.

Sea level forecast is obtained using a set of 50 ECMWF perturbed mean SL pressure forecasts and $10 \mathrm{~m}$ wind forecast to force hydrodynamic model HYPSE [10] (see for the domain Figure 1) and producing the corresponding set of EPS forecasts in the Adriatic Sea. Then, a simple procedure is developed in order to compute the forecast uncertainty without performing the hydrodynamic model simulations. 
The hydrodynamic model computes the sea residual (SR), which is the SL after the subtraction of the astronomical tide, including exclusively the superposition of storm surge and seiches. This is justified because of the modest tidal range in the Adriatic Sea, which makes negligible nonlinear interactions with the surge, allowing computing astronomical tides and SR independently and then superimposing them, introducing negligible errors.

The perturbed SR forecasts are used for representing the uncertainty of the standard high resolution deterministic forecast (DF), neglecting errors due to inaccuracy of the hydrodynamic model and the uncertainty in the initial SR condition.

Forcing resolution of the meteorological fields is T1279 and T639 for the DF and the ECMWF EPS fields, respectively. These, downloaded at 0.125 degs (DF) and 0.25 degs (EPS), have been linearly interpolated to the HYPSE grid, which is the same for all $50+1$ simulations.

The hydrodynamic model used in this study adopts a rectangular mesh grid of variable size in the range from 3.3 to $7 \mathrm{~km}$. The grid step starts from 0.03 degrees in the northern part of the Adriatic Sea, increasing with a factor of 1.01 in both latitude and longitude. This grid has been used in a previous study [10], producing more accurate results with respect to other grids. The domain contains a unique open boundary in the Otranto Strait, across which a fixed sea level is imposed. The model does account neither for changes of total water mass of the Adriatic Sea nor for steric effects on the water surface. This limitation is compensated by a bias removal technique [17], which takes into account the effect of long term (several days to month) variability of the mean SL in the Adriatic Sea. Figure 1 shows HYPSE domain and the location of tide and pressure gauges used in the present work.

In the three months' period, the $50+1$ HYPSE runs are carried out twice per day, forced by the ECMWF meteorological fields at 00:00 and 12:00 UTC. Each run, which uses the same version of the HYPSE model, provides a 6-day forecast and is initialized by a ten-day analysis run, where HYPSE is driven by the high resolution (T1279) ECMWF analysis, in order to have the same initial in all $50+1$ SR predictions.

This EPS forecast set is complemented with a hindcast (initialized with a 10-day-long analysis) covering exactly the same period in which the same version of the HYPSE model has been driven by the ECMWF analysis. The hindcast performs better than the DF, suggesting errors in the meteorological prediction of the conditions leading to the onset of the storm surge [17].

The spread of the 50 EPS members around their mean provides an estimate of the forecast uncertainty. For any selected threshold, the fraction of EPS members above it at a given time provides an estimate of the probability of crossing it in the future evolution of the SL [18], a fundamental tool to properly manage the MOSE barriers.

Estimates of the forecast uncertainty can be alternatively obtained using the ensemble dressing forecast (EDF) technique. It consists in the aggregation between DF and a prescribed uncertainty, which is computed on the basis of the spread of the meteorological fields and the overall DF error [24]. This method is useful to avoid running 50 EPS members and thus to reduce the computational cost of the probabilistic forecast. Previous studies [24] have shown that the uncertainty that is estimated by a linear combination of suitable meteorological variances approximates well that obtained using the EPS. Here, this computationally cheaper method has been used to produce a probability forecast by combining the DF with the prescribed (Gaussian) probability distribution that is obtained from both the meteorological fields spread and the DF overall error, which is proportional to ensemble member spread.

This new method is called "multiple probabilistic dressed forecast" (MULTIPDF). It assumes a Gaussian probability distribution:

$$
P(h)=\frac{1}{\sigma \sqrt{2 \pi}} e^{-\left(h-h_{\mathrm{DF}}(t)\right) / 2 \sigma^{2}},
$$

where $h$ is the sea level and $h_{\mathrm{DF}}(t)$ is the value predicted by the deterministic forecast. The standard deviation $\sigma$ is parametrized as the sum of two terms:

$$
\sigma=\alpha(t) A+\beta(t) B, \quad \text { where } \alpha(t)+\beta(t)=1 .
$$

This is a weighted mean, with different coefficients $\alpha(t)$ and $\beta(t)$ for increasing lead times $t$, of term $A$ and term $B$.

The first term, $A$, represents the EPS uncertainty. It is the overall DF rms error (strictly related to the EPS spread [17]) for corresponding gauge, lead time, and DF magnitude (data are aggregated with a $10 \mathrm{~cm}$ SR step), describing the increasing of uncertainty with the SR level and lead time:

$$
A=\text { rms error (gauge, } t, \text { DF magnitude). }
$$

The second term, $B$, represents the meteorological uncertainty. It is a linear combination, of the variances of four atmospheric pressure gradients in the Mediterranean Sea that are used in the ICPSM surge forecast statistical models [9, 24, 25]. These gradients are suitable to describe the meteorological fields uncertainty due to the strong correlation between them and the SR. These gradients are computed between CNR platform and Bari, CNR platform and Zara, and CNR platform and Genova and between Genova and Porto Torres (Figure 1). Their variance is slightly different for each gauge, but it does not depend on the storm surge level and forecast lead time:

$$
\begin{aligned}
B= & a \cdot \mathrm{rms} \Delta P_{\mathrm{CNR}_{\text {Bari }}}+b \cdot \operatorname{rms} \Delta P_{\mathrm{CNR}_{\text {Zara }}} \\
& +c \cdot \mathrm{rms} \Delta P_{\mathrm{CNR}_{\text {Genova }}}+d \cdot \mathrm{rms} \Delta P_{\mathrm{Genovap}_{\text {orto Torres }}} .
\end{aligned}
$$

The contribution of coefficients $a, b, c$, and $d$ is computed via a Leave-One-Out Cross Validation procedure [26]. This method predicts the property value for a compound from the data set, which is in turn predicted from the regression equation calculated from the data for all other compounds. The procedure has been applied over the 20 greatest storm surges, in order to check for overfitting: the coefficients have been calibrated considering 19 storm surges and a prediction was made for the 20th; this procedure has been applied for each event. The Leave-One-Out Cross Validation procedure produces twenty different estimates of the coefficients values, 


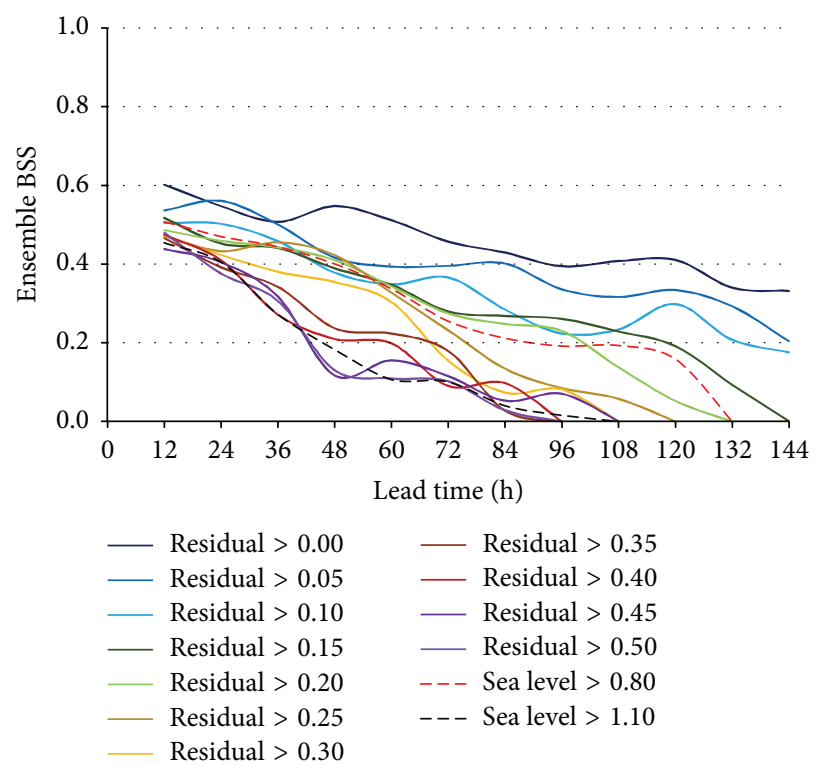

(a)

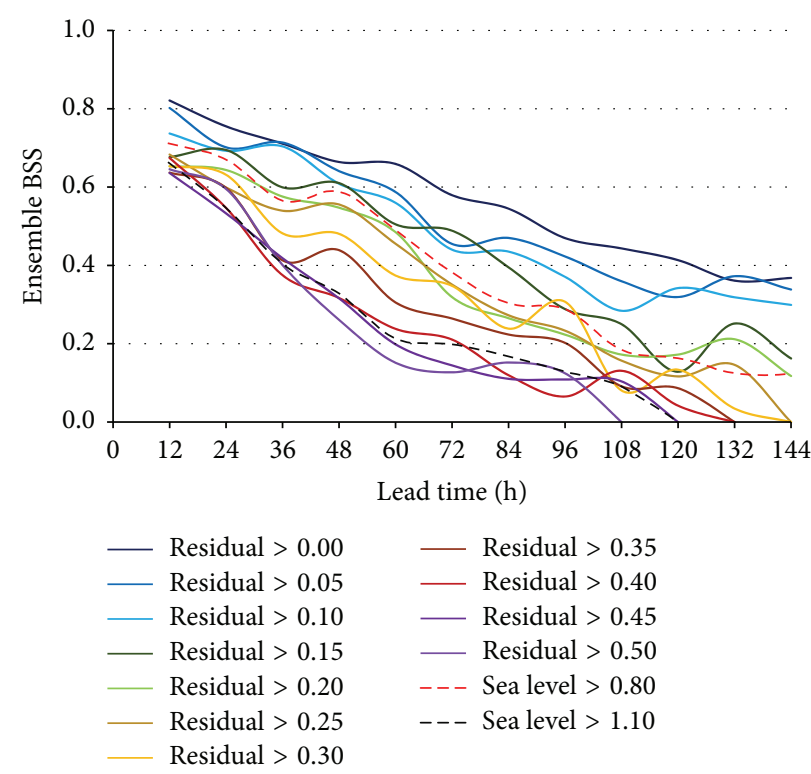

(b)

FIGURE 2: Brier skill score as function of lead time for the eleven SR thresholds (continuous lines) and the two SL thresholds (dash lines). In (a)/(b), the BSS is computed with respect to observations/hindcast.

with standard error lower than $10 \%$. Their mean has been adopted as actual value.

Finally, the weight of terms $A$ and $B$, regarding the overall DF rms error and the variance of the pressure gradients and expressed through coefficients $\alpha(t)$ and $\beta(t)$, has been found in order to optimize the MULTIPDF via simple linear regression. Results show an almost linear trend of the weight in function of the lead time: for lead time of 12 hours we have found weight of 0.8 and 0.2 for the variance of the pressure gradients and the overall DF rms error, respectively, and vice versa for lead time of 144 hours.

\section{Results and Discussion}

If we look at the most important storm surges, there are little differences between the ensemble members and observed $\mathrm{SR}$, in particular for small lead times, where the majority of the ensembles capture the peaks and the majority of the observations lay inside the ensemble forecast distribution; this suggests that the EPS probabilistic forecast is reliable.

The ensemble dressing method will be considered successful if it produces results comparable to the EPS probabilistic forecast. The Brier score and Brier skill score [27] are the indexes commonly used in measures for evaluating the accuracy of probability forecasts [28]. The Brier skill score (BSS) is first computed on the basis of the EPS as function of the lead time for eleven different SR thresholds (from 0 to $0.5 \mathrm{~m}$ ) and two SL alert and alarm thresholds, equal to 0.8 and $1.1 \mathrm{~m}$ over the benchmark of Punta Salute gauge, corresponding to this day to mean sea level recorded in 1987. Data are aggregated considering five gauges, four real gauges along the Adriatic Sea (Trieste, Rovinj, Split, and Dubrovnik) and a fifth virtual gauge given by the mean of the levels at the three lagoon inlets (Figure 1). Results show that the EPS BSS remains positive for very large lead times and it diminishes for increasing thresholds (Figure 2).

EDF aims to provide a simplified estimate of forecast uncertainty. Four alternative dressings are presented, providing different ways to choose the width of the Gaussian distribution.

The first is an undressed probability forecast (UDPF) that assumes a perfect forecast with no uncertainty. This gives probability 1.0 when the DF is above the threshold, with 0.0 otherwise (this can be considered a limit case in which a Gaussian with zero standard deviation is adopted). The second is an error dressed probabilistic forecast (EDPF) that assumes a fixed width centred on the DF with a prescribed standard deviation corresponding to the overall (gauges and lead times) rms error of the DF. The third is the meteo-dressed probabilistic forecast (MDPF), which assumes a width equal to a linear normalized combination of the variances of four suitable pressure gradients in the Mediterranean Sea.

The final variant, MULTIPDF, as described in Section 2, is the best kind of forecast dressing that allows estimating adequately the EPS spread.

The four dressed forecasts are compared for the SR threshold of 0,25 , and $50 \mathrm{~cm}$ and for the SL threshold of 80 (alert) and 110 (alarm) cm (Figure 3), providing a benchmark for the EPS performance and illustrating the importance of different aspects of uncertainty.

Figure 3 shows the application of the ensemble dressing method during the selected months. To capture the forecast uncertainty, the DF has been dressed with four different Gaussian distributions.

Results show that EPS probabilistic forecast slightly outperforms the dressing methods score for almost every threshold and lead time, especially in the UDPF. For SR $=0 \mathrm{~m}$, the BSS index of all other dressing methods remains consistently for a 5-day-long period above 0.2 and 0.4 with respect to 


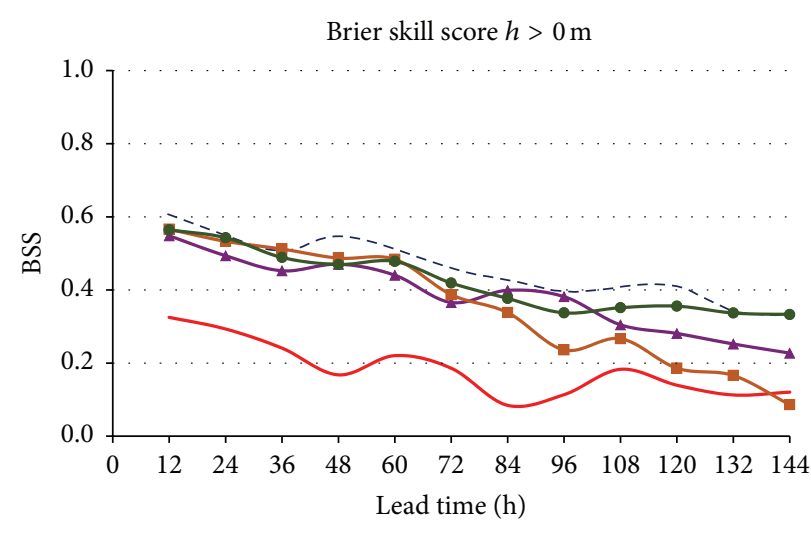

(a)

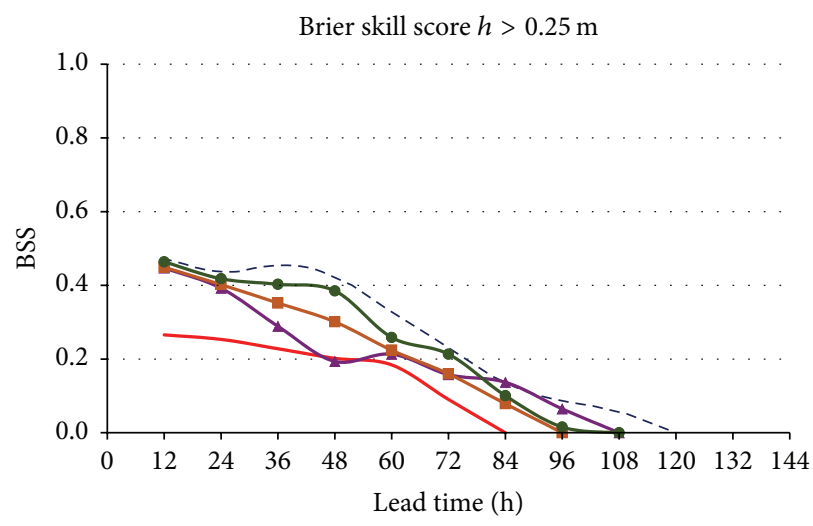

(c)

Brier skill score $h>0.50 \mathrm{~m}$

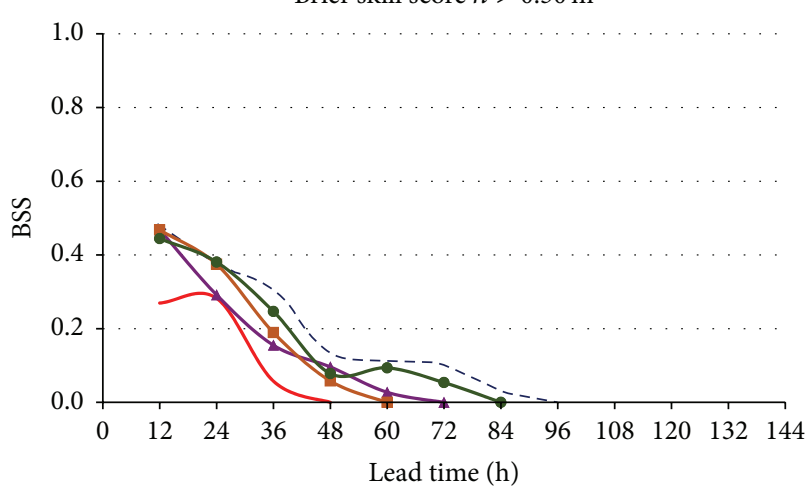

(e)

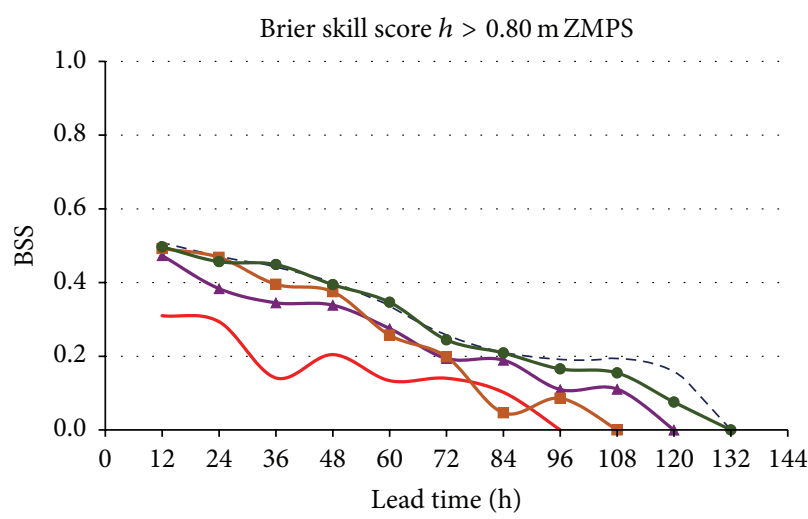

(g)

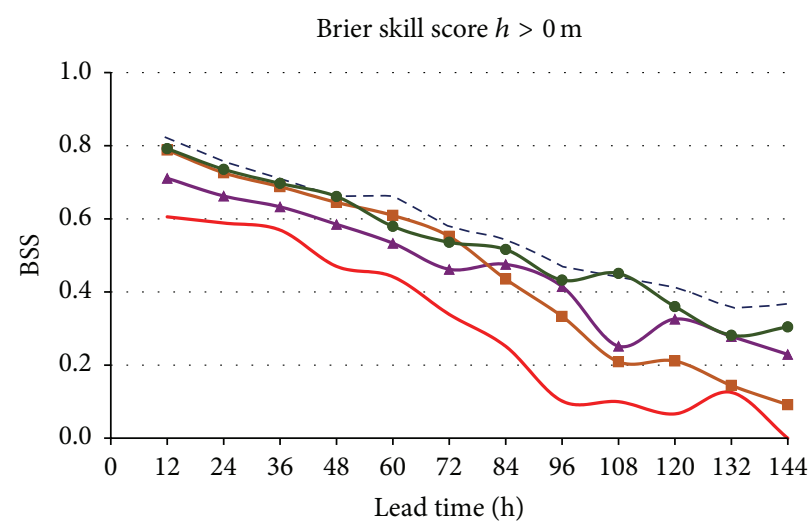

(b)

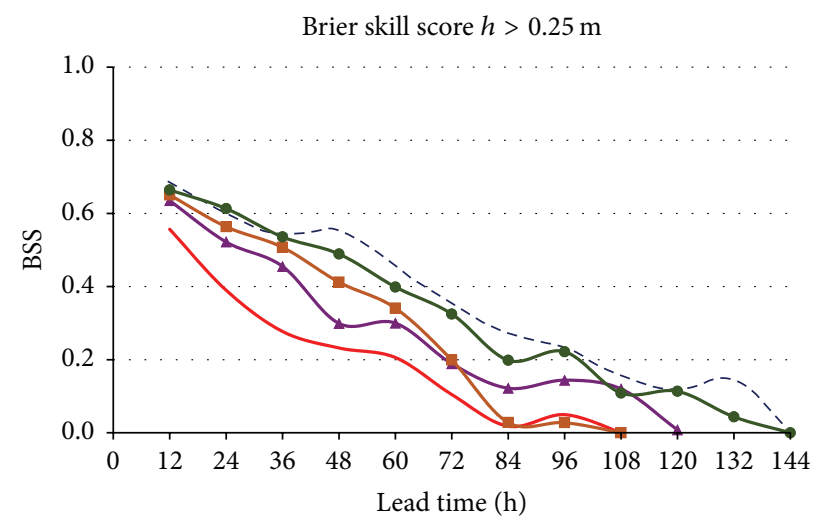

(d)

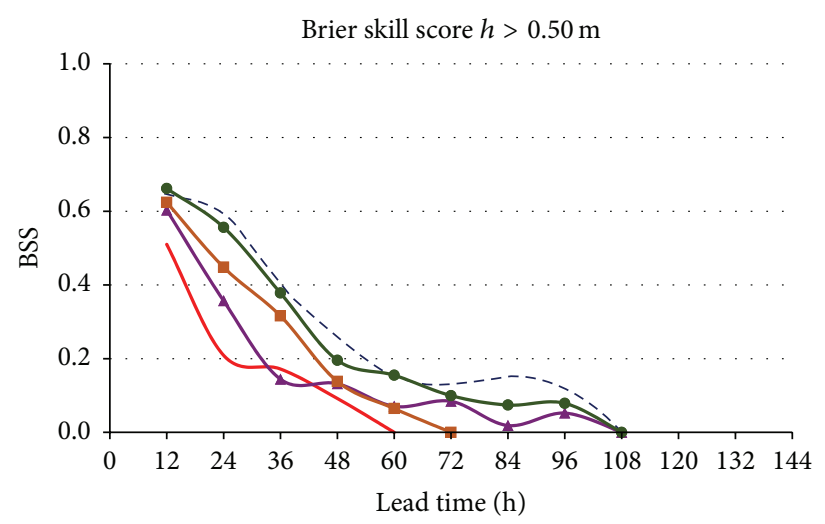

(f)

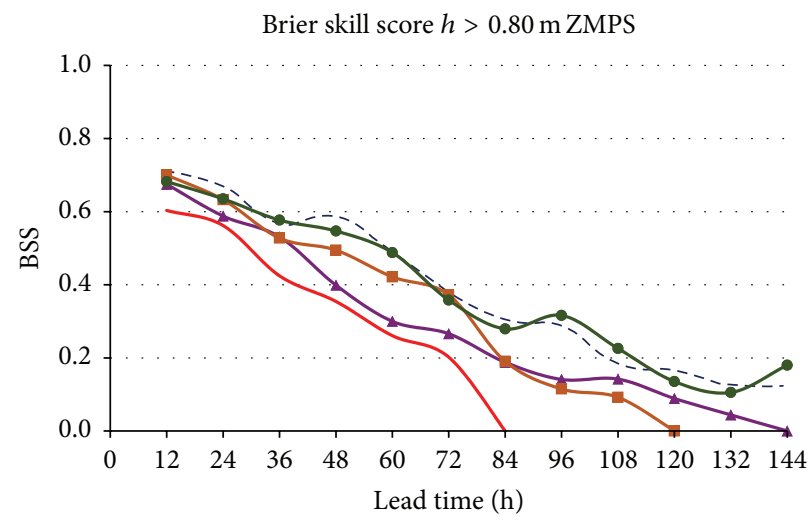

(h)

Figure 3: Continued. 


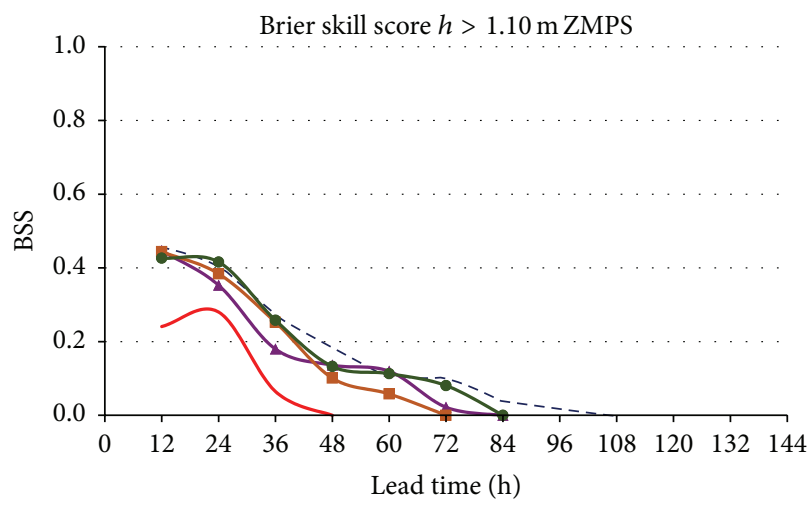

(i)

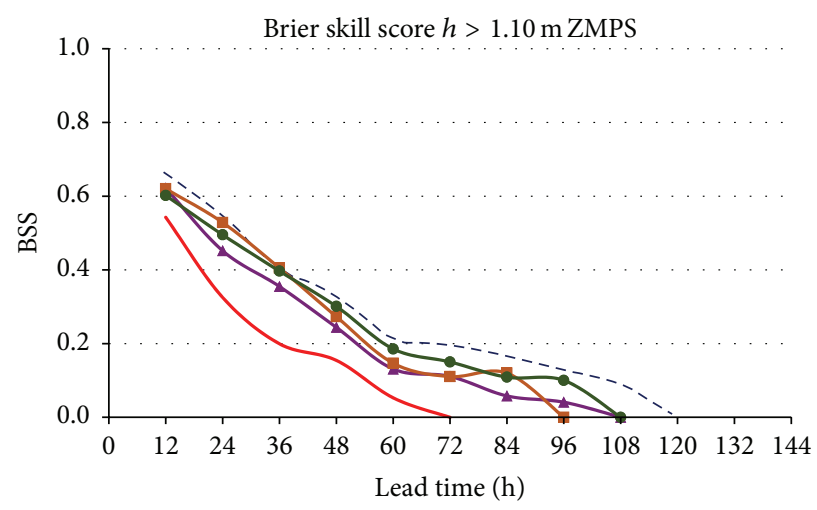

(j)

FIGURE 3: Brier skill score as function of lead time for the four different dressing methods (red thick line: UDPF, violet continuous cum dotted line: EDPF, brown continuous cum dotted line: MDPF, and green continuous cum dotted line: MULTIPDF) is compared to the EPS BSS (blue dash line). In (a, c, e, g, i )/(b, d, f, h, j), the BSS is computed with respect to observations/hindcast, considering SR $=0,25$, and $50 \mathrm{~cm}$ and $\mathrm{SL}$ $=80$ and $110 \mathrm{~cm}$.

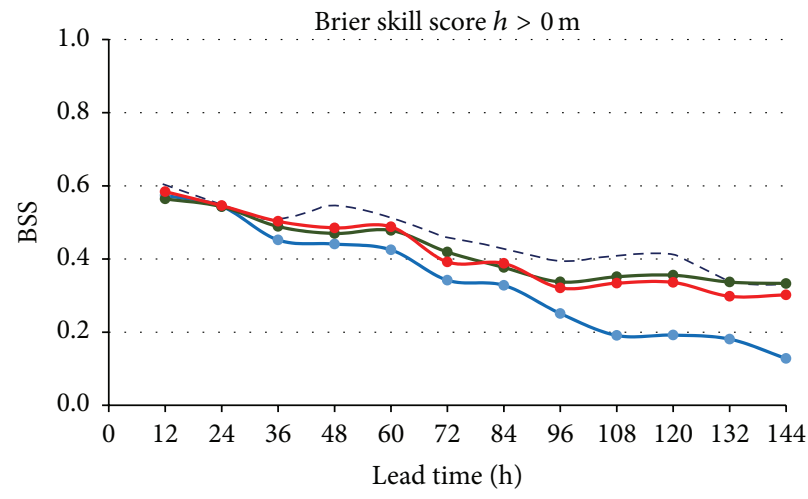

(a)

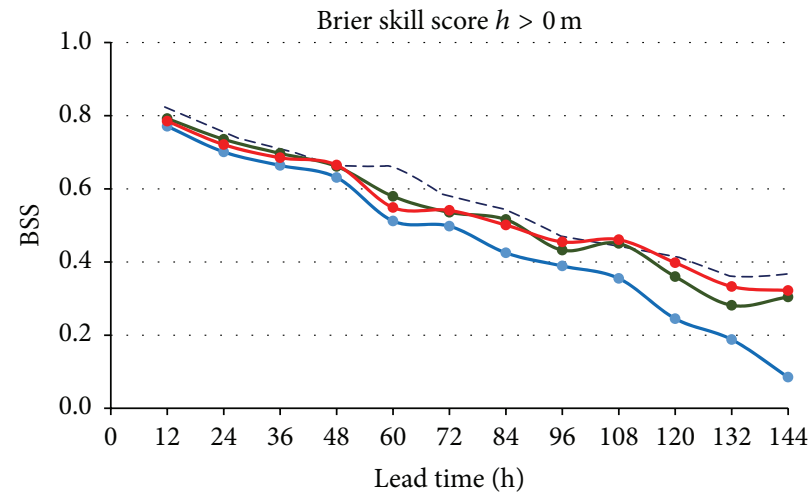

(b)

FIGURE 4: Brier skill score as function of lead time for the MULTIPDF dressing method applied to three different forecasts (green continuous cum dotted line: DF, red continuous cum dotted line: EMF, and blue continuous cum dotted line: CRF) is compared to the EPS BSS (blue dash line). In (a)/(b), the BSS index is computed with respect to observations/hindcast, considering SR = zero.

observation and hindcast, respectively, while UDPF index drops below 0.2/0.4 already after three days (Figures 3(a) and $3(b)$ ). Figures $3(i)$ and $3(j)$ show that, considering the SL alarm threshold equal to $1.1 \mathrm{~m}$, fundamental for the MOSE barriers management, the UDPF BSS drops below 0 after 48 hours with respect to observations (after 72 hours with respect to the hindcast). This is a strong indication that to obtain a reliable probability estimate using only the DF is not possible. EDPF and MDPF present a better performance, in particular for little and long lead times, respectively. This is an important result, showing that for lead times lower than 2-3 days the majority of uncertainty can be described by the spread of the meteorological fields. For higher lead times, EDPF performs better than MDPF, due to the unpredictability of wind speed and direction. Finally, the MULTIPDF, which accounts for uncertainty contribution for all gauges and lead times, provides a higher BSS index than other dressing methods. Therefore, it could be used as a cheap alternative to the expensive EPS probabilistic forecast.
These results are obtained by the application of four ensemble dressing methods to the DF. The same methods can be used to dress the mean of the ensemble members (ensemble mean forecast, EMF) and the control run (control run forecast, CRF), without significant improvements. Figure 4 shows an example of the BSS index for SR greater than zero, comparing the performances of the MULTIPDF applied to DF, EMF, and CRF, respectively. Results show that EMF and DF have a comparable reliability, slightly higher (lower) for DF than for EMF at short (long) lead times.

The MULTIPDF is a method that attempts to provide the best dressed forecast. Many realizations of dressing parameters have been computed in order to improve the performance of the dressed forecasts, finding a linear combination between the overall rms error and the meteorological fields spread. However, a Gaussian distribution, while simple, could not be the best fit for the EPS error distribution. The results are meant to illustrate the complexities of producing probabilistic forecasts based only on historic statistics of climatology 
and EPS performance. By contrast, the MULTIPDF well simulates the ensemble uncertainty and although it should be more robust and adaptable, the key aspects have been found accurately enough to be applied to real-time surge forecast and MOSE barriers management.

The results, presented using the aggregated data for all the gauges, differ very slightly if a single gauge is analyzed, as in [18], suggesting the good performance of the proposed method to describe the Adriatic Sea uncertainty.

\section{Conclusions}

A fully informative and accurate storm surge forecast is of paramount importance for an effective warning of the population in Venice and in the other islands of the lagoon, reducing damage produced by sea hazards. This contribution describes an improved method that includes meteorological uncertainty in single deterministic forecasting applications, in order to improve the information contained in real-time forecast for the city of Venice and its lagoon.

Ensemble dressing forecast has been shown to be a fast method to produce a probabilistic forecast, without performing the EPS simulations. The novelty of this study consists in the form of the multiple probabilistic dressed forecast (called MULTIPDF) that has been used; MULTIPDF is obtained by dressing the DF with a prescribed Gaussian distribution, whose variance accounts for many factors. It depends on the overall DF rms error, it is function of the forecast lead time and the surge magnitude of the meteorological uncertainty, expressed via linear combination of suitable pressure gradients, and it is a function of the tide gauge. Therefore, MULTIPDF is meant to be a practical computationally cheap alternative to EPS forecast.

The methodology presented in this contribution was kept purposefully simple and intuitive, and many extensions, variants, and improvements are possible according to both the improvement of the EPS forecast and the coupling between EDF and data assimilation methods. One limitation of EDF is that it is unable to correct situations in which the EPS is underconfident. Moreover, the Gaussian distribution does not always adequately fit the EPS spread. Finally, like most of postprocessing methods, ensemble dressing method is developed for single gauges, not considering spatial correlation between the different locations.

The MULTIPDF ensemble dressing method performs well at all the tide gauges considered in this study and it can be an effective tool to integrate the information of the deterministic storm surge forecast for the Venice Lagoon in the future, when the MOSE barriers are operational. However, it may be expected that MULTIPDF ensemble dressing is not able to reproduce eventual outliers in very nonlinear conditions and that it underestimates uncertainty in cases when the meteorological forecast is very unstable with respect to errors in its initialization.

\section{Acronyms}

BSS: Brier skill score

CRF: Control run forecast

$\begin{array}{ll}\text { DF: } & \text { Deterministic (high resolution) forecast } \\ \text { EDF: } & \text { Ensemble dressing forecast } \\ \text { EDPF: } & \text { Error dressed probabilistic forecast } \\ \text { EMF: } & \text { Ensemble mean forecast } \\ \text { EPS: } & \text { Ensemble prediction system } \\ \text { MDPF: } & \text { Meteo-dressed probabilistic forecast } \\ \text { MULTIPDF: } & \text { Multiple probabilistic dressed forecast } \\ \text { SL: } & \text { Sea level } \\ \text { SR: } & \text { Sea residual } \\ \text { UDPF: } & \text { Undressed probabilistic forecast. }\end{array}$

\section{Competing Interests}

The authors declare that they have no competing interests.

\section{Acknowledgments}

The hourly SL observations in CNR PLATFORM and Venice "Punta Salute" were provided by ICPSM of the Venice City Council, observations in Trieste by Dr. Fabio Raicich of ISMAR-CNR (Italy), and observations in Rovinj, Split, and Dubrovnik by Dr. Nenad Leder of the Croatian Hydrographic Institute.

\section{References}

[1] R. J. Nicholls, P. P. Wong, V. R. Burkett et al., "Coastal systems and low-lying areas," in Climate Change 2007: Impacts, Adaptation and Vulnerability, R. J. Nicholls, P. P. Wong, V. R. Burkett et al., Eds., pp. 315-356, Cambridge University Press, Cambridge, UK, 2007.

[2] M. Verlaan, A. Zuderveld, H. De Vries, and J. Kroos, "Operational storm surge forecasting in the Netherlands: developments in the last decade," Philosophical Transactions of the Royal Society A: Mathematical, Physical and Engineering Sciences, vol. 363, no. 1831, pp. 1441-1453, 2005.

[3] E. M. Lane, R. A. Walters, P. A. Gillibrand, and M. Uddstrom, "Operational forecasting of sea level height using an unstructured grid ocean model," Ocean Modelling, vol. 28, no. 1-3, pp. 88-96, 2009.

[4] A. R. Robinson, A. Tomasin, and A. Artegiani, "Flooding of Venice: phenomenology and prediction of the Adriatic storm surge," Quarterly Journal of the Royal Meteorological Society, vol. 99, no. 422, pp. 688-692, 1973.

[5] P. Lionello, "Extreme storm surges in the gulf of Venice: present and future climate," in Venice and Its Lagoon, State of Knowledge, C. Fletcher and T. Spencer, Eds., pp. 59-65, 2005.

[6] P. Lionello, R. Mufato, and A. Tomasin, "Sensitivity of free and forced oscillations of the Adriatic Sea to sea level rise," Climate Research, vol. 29, no. 1, pp. 23-39, 2005.

[7] P. Lionello, L. Cavaleri, K. M. Nissen, C. Pino, F. Raicich, and U. Ulbrich, "Severe marine storms in the Northern Adriatic: characteristics and trends," Physics and Chemistry of the Earth, vol. 40-41, pp. 93-105, 2012.

[8] P. Lionello, "The climate of the Venetian and North Adriatic region: variability, trends and future change," Physics and Chemistry of the Earth, vol. 40-41, pp. 1-8, 2012.

[9] P. Canestrelli and F. Moretti, "I modelli statistici del comune di Venezia per la previsione della marea: valutazioni e confronti sul 
quinquennio 1997-2001," in Atti Dell'Istituto Veneto di Scienze Lettere ed Arti, pp. 479-516, Tomo CLXII, 2004.

[10] P. Lionello, A. Sanna, E. Elvini, and R. Mufato, "A data assimilation procedure for operational prediction of storm surge in the northern Adriatic Sea," Continental Shelf Research, vol. 26, no. 4, pp. 539-553, 2006.

[11] N. van Velzen and M. Verlaan, "COSTA a problem solving environment for data assimilation applied for hydrodynamical modelling," Meteorologische Zeitschrift, vol. 16, no. 6, pp. 777793, 2007.

[12] K. Beven, "Changing ideas in hydrology-the case of physicallybased models," Journal of Hydrology, vol. 105, no. 1-2, pp. 157$172,1989$.

[13] H. K. McMillan and J. Brasington, "End-to-end flood risk assessment: a coupled model cascade with uncertainty estimation," Water Resources Research, vol. 44, no. 3, Article ID W03419, 2008.

[14] L. Zhong, M. Li, and D.-L. Zhang, "How do uncertainties in hurricane model forecasts affect storm surge predictions in a semi-enclosed bay?" Estuarine, Coastal and Shelf Science, vol. 90, no. 2, pp. 61-72, 2010.

[15] J. Flowerdew, K. Horsburgh, C. Wilson, and K. Mylne, "Development and evaluation of an ensemble forecasting system for coastal storm surges," Quarterly Journal of the Royal Meteorological Society, vol. 136, no. 651, pp. 1444-1456, 2010.

[16] J. Flowerdew, K. Mylne, C. Jones, and H. Titley, "Extending the forecast range of the UK storm surge ensemble," Quarterly Journal of the Royal Meteorological Society, vol. 139, no. 670, pp. 184-197, 2013.

[17] R. Mel and P. Lionello, "Storm surge ensemble prediction for the city of Venice," Weather and Forecasting, vol. 29, no. 4, pp. 1044-1057, 2014.

[18] R. Mel and P. Lionello, "Verification of an ensemble prediction system for storm surge forecast in the Adriatic Sea," Ocean Dynamics, vol. 64, no. 12, pp. 1803-1814, 2014.

[19] A. J. Condon, Y. P. Sheng, and V. A. Paramygin, "Toward highresolution, rapid, probabilistic forecasting of the inundation threat from landfalling hurricanes," Monthly Weather Review, vol. 141, no. 4, pp. 1304-1323, 2013.

[20] R. Dawson, J. Hall, P. Sayers, P. Bates, and C. Rosu, "Samplingbased flood risk analysis for fluvial dike systems," Stochastic Environmental Research and Risk Assessment, vol. 19, no. 6, pp. 388-402, 2005.

[21] T. C. Pagano, D. L. Shrestha, Q. J. Wang, D. Robertson, and P. Hapuarachchi, "Ensemble dressing for hydrological applications," Hydrological Processes, vol. 27, no. 1, pp. 106-116, 2013.

[22] V. Fortin, A.-C. Favre, and M. Saï, "Probabilistic forecasting from ensemble prediction systems: improving upon the bestmember method by using a different weight and dressing kernel for each member," Quarterly Journal of the Royal Meteorological Society, vol. 132, no. 617, pp. 1349-1369, 2006.

[23] J. Brcker and A. S. Leonard, "From ensemble forecasts to predictive distribution functions," Tellus A, vol. 60, no. 4, pp. 663-678, 2008.

[24] R. Mel, D. P. Viero, L. Carniello, A. Defina, and L. D’Alpaos, "Simplified methods for real-time prediction of storm surge uncertainty: the city of Venice case study," Advances in Water Resources, vol. 71, pp. 177-185, 2014.

[25] G. Umgiesser, D. M. Canu, A. Cucco, and C. Solidoro, "A finite element model for the Venice Lagoon. Development, set up, calibration and validation," Journal of Marine Systems, vol. 51, no. 1-4, pp. 123-145, 2004.
[26] M. Stone, "An asymptotic equivalence of choice of model by cross-validation and Akaike's criterion," Journal of the Royal Statistical Society, Series B: Methodological, vol. 39, no. 1, pp. 4447, 1977.

[27] G. W. Brier, "Verification of forecasts expressed in terms of probability," Monthly Weather Review, vol. 78, no. 1, pp. 1-3, 1950.

[28] M. S. Roulston, "Performance targets and the Brier score," Meteorological Applications, vol. 14, no. 2, pp. 185-194, 2007. 

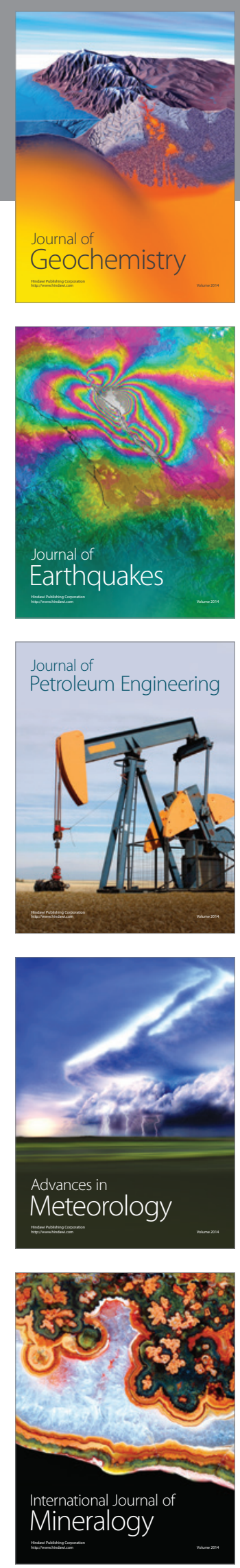
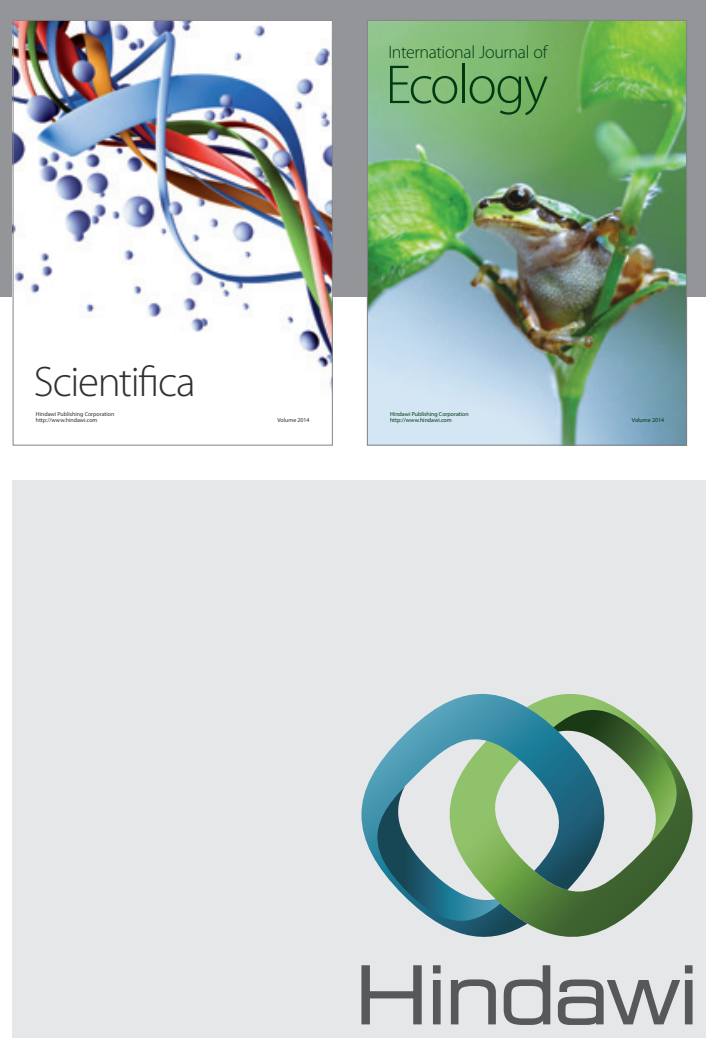

Submit your manuscripts at

http://www.hindawi.com
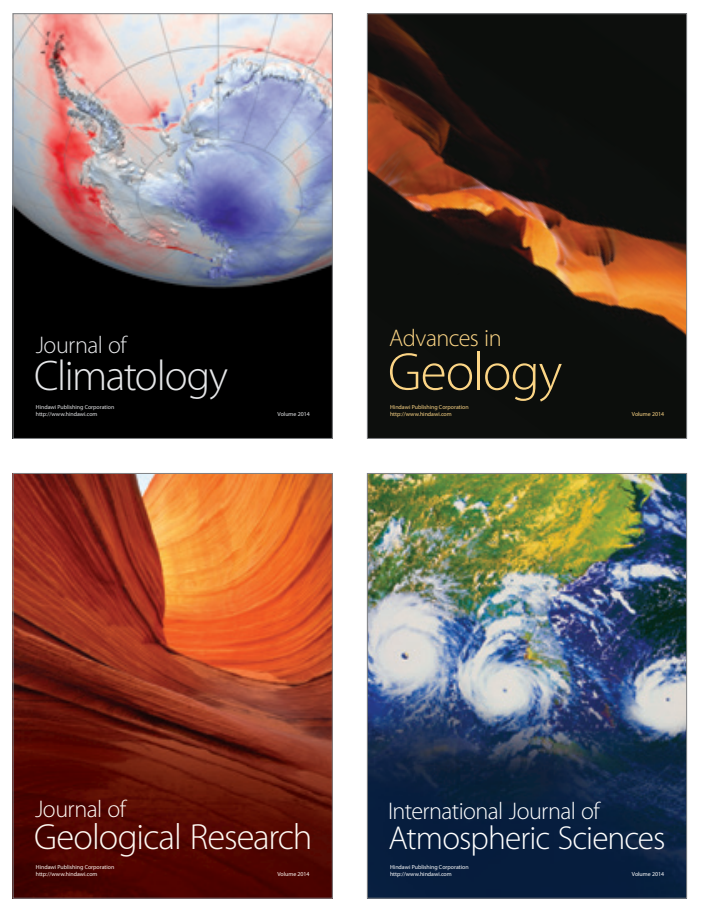

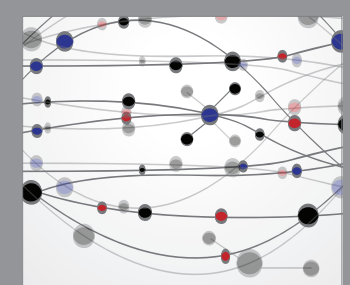

The Scientific

\section{World Journal}
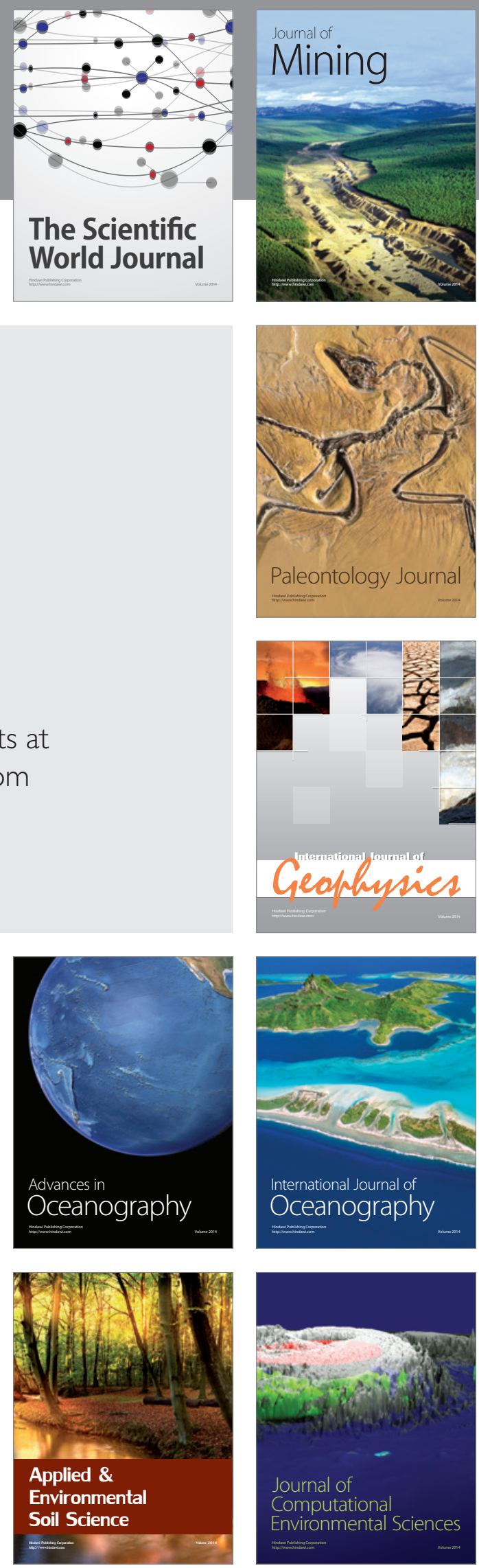